\title{
ARTICLE
}

Molecular targets for therapy

\section{A novel CDK9 inhibitor increases the efficacy of venetoclax (ABT-199) in multiple models of hematologic malignancies}

\author{
Darren C. Phillips ${ }^{1}$. Sha Jin ${ }^{1}$ - Gareth P. Gregory $\mathbb{1}^{2,3} \cdot$ Qi Zhang $^{4} \cdot$ John Xue $^{1} \cdot$ Xiaoxian Zhao $^{5}$ - Jun Chen ${ }^{6}$. \\ Yunsong Tong ${ }^{1} \cdot$ Haichao Zhang $^{1}$ - Morey Smith ${ }^{1}$. Stephen K. Tahir ${ }^{1} \cdot$ Rick F. Clark $^{1}$ - Thomas D. Penning ${ }^{1}$. \\ Jennifer R. Devlin ${ }^{2,7}$ - Jake Shortt ${ }^{3}$ - Eric D. Hsi ${ }^{5}$ - Daniel H. Albert ${ }^{1}$ - Marina Konopleva ${ }^{4} \cdot$ Ricky W. Johnstone $^{2,7}$. \\ Joel D. Leverson ${ }^{8} \cdot$ Andrew J. Souers $^{1}$
}

Received: 27 November 2018 / Revised: 18 October 2019 / Accepted: 13 November 2019 / Published online: 11 December 2019

(c) The Author(s) 2019

\begin{abstract}
MCL-1 is one of the most frequently amplified genes in cancer, facilitating tumor initiation and maintenance and enabling resistance to anti-tumorigenic agents including the BCL-2 selective inhibitor venetoclax. The expression of MCL-1 is maintained via P-TEFb-mediated transcription, where the kinase CDK9 is a critical component. Consequently, we developed a series of potent small-molecule inhibitors of CDK9, exemplified by the orally active A-1592668, with CDK selectivity profiles that are distinct from related molecules that have been extensively studied clinically. Short-term treatment with A-1592668 rapidly downregulates RNA pol-II (Ser 2) phosphorylation resulting in the loss of MCL-1 protein and apoptosis in MCL-1dependent hematologic tumor cell lines. This cell death could be attenuated by either inhibiting caspases or overexpressing BCL-2 protein. Synergistic cell killing was also observed between A-1592668 or the related analog A-1467729, and venetoclax in a number of hematologic cell lines and primary NHL patient samples. Importantly, the CDK9 inhibitor plus venetoclax combination was well tolerated in vivo and demonstrated efficacy superior to either agent alone in mouse models of lymphoma and AML. These data indicate that CDK9 inhibitors could be highly efficacious in tumors that depend on MCL-1 for survival or when used in combination with venetoclax in malignancies dependent on MCL-1 and BCL-2.
\end{abstract}

\section{Introduction}

Cyclin-dependent kinases (CDKs) are serine-threonine kinases that function to coordinate multiple cellular

Supplementary information The online version of this article (https:// doi.org/10.1038/s41375-019-0652-0) contains supplementary material, which is available to authorized users.

$\triangle$ Darren C. Phillips

darren.phillips@abbvie.com

1 Oncology-Discovery, AbbVie Inc., 1 North Waukegan Road, North Chicago, IL 60064, USA

2 Peter MacCallum Cancer Centre, Translational Hematology Program, 305 Grattan Street, Melbourne, VIC 3000, Australia

3 Blood Cancer Therapeutics Laboratory, School of Clinical Sciences at Monash Health, Monash University, 246 Clayton Rd, Clayton, VIC 3168, Australia

4 Department of Leukemia, The University of Texas MD Anderson Cancer Center, 1515 Holcombe Blvd, Houston, TX 77030, USA functions. Whereas $\mathrm{CDKs} 1,2$, 4, and 6 are primarily involved in the regulation of the cell cycle, CDK7, CDK8, and CDK9 play a role in regulating transcription to further influence cell proliferation and survival by driving the expression of numerous target genes. Consequently, aberrant expression of various CDKs results in the loss of proliferative control and/or enhanced survival potential, phenotypes considered as hallmarks of cancer [1, 2]. The relevance of CDKs to cancer growth and survival has

5 Department of Laboratory Medicine, Robert J. Tomsich Pathology and Laboratory Medicine Institute, Cleveland Clinic, Cleveland, OH 44195, USA

6 TEST, AbbVie Inc., 1 North Waukegan Road, North Chicago, IL 60064, USA

7 The Sir Peter MacCallum Department of Oncology, The University of Melbourne, Grattan Street, Parkville, VIC 3052, Australia

8 Oncology-Development, AbbVie Inc., 1 North Waukegan Road, North Chicago, IL 60064, USA 
garnered widespread interest in the generation of CDK inhibitors, several of which have been evaluated for efficacy in human tumors [3]. Flavopiridol has shown objective responses in CLL $[4,5]$ and other hematologic malignancies $[6,7]$ and is exemplary of how the CDK inhibitor field has evolved. This, and the majority of early CDK inhibitors were initially thought to drive antiproliferative activity via cell cycle inhibition; however, investigational and clinical studies ultimately indicated that antitumor efficacy was driven at least partially though inhibition of transcriptional kinases including CDK9 [3].

CDK9 is the catalytic subunit of the cyclin T1/CDK9 complex, P-TEFb. CDK9 phosphorylates serine-2 within heptapeptide repeats on the C-terminal domain of RNA polymerase-II (RNA pol-II) to initiate its release into the elongation phase of gene transcription [8, 9]. Prominent oncogenes regulated by CDK9 include but are not limited to $M Y C$ and MCL1, the latter of which encodes a member of the BCL-2 family of proteins that regulate apoptosis. MCL1, along with BCL-2, BCL- $\mathrm{X}_{\mathrm{L}}, \mathrm{BCL} 2-\mathrm{A} 1$, and BCL-W, comprises the antiapoptotic sub-group that counter the activity of the pro-apoptosis proteins, which are further subdivided into the $\mathrm{BH} 3$-only proteins such as $\mathrm{BIM}$ and $\mathrm{BAD}$, and the multi-BH domain effectors BAX, BAK, and BOK. The proapoptotic members can be sequestered by direct protein-protein interactions via the $\mathrm{BH} 3$-binding groove contained within the antiapoptotic BCL-2 family members. The aberrant expression and/or function of the BCL-2 family proteins facilitates the development and persistence of malignancies, enabling cancer cells to survive "stresses" that ordinarily result in apoptosis $[10,11]$.

Over-reliance on a single antiapoptotic protein to maintain survival facilitates therapeutic exploitation and a number of small-molecule inhibitors that target the antiapoptotic BCL-2 family members are in various stages of clinical development [12]. The most advanced of these, venetoclax (ABT-199) [13], first received FDA approval in the USA for use in patients with relapsed/refractory $(\mathrm{R} / \mathrm{R})$ $17 p$-deleted CLL, an indication characterized by high BCL2 expression [14, 15]. Although venetoclax administration has demonstrated clinical efficacy in $\mathrm{R} / \mathrm{R}$ non-Hodgkin lymphoma (NHL) and acute myelogenous leukemia (AML) patients, the responses in these settings are not of the same magnitude as those observed in R/R CLL (ORR of 79\%) [16], highlighting the potential role of additional survival factors. MCL-1 has been demonstrated to play a role in both inherent and acquired resistance to venetoclax in numerous preclinical models of hematologic malignancies [17-19]. Agents capable of targeting MCL-1 directly or indirectly would therefore have the potential to increase the activity of venetoclax in various hematological malignancies.

The anti-tumorigenic activity of flavopiridol or dinaciclib in tumor cells is at least partially attributed to the rapid induction of apoptosis that results from CDK9 inhibition. This is particularly apparent in tumor cell lines that are dependent upon MCL-1 for survival, where the abrogation of transcription leads to a rapid reduction in this and other short-lived survival factors following short-term drug exposure [20-25]. Among CDK9 inhibitors, flavopiridol has been studied most extensively in the clinic; while trials with this agent have shown encouraging clinical efficacy, a complex toxicity profile associated with activity beyond CDK9 has hampered development [3]. The consequence of this promiscuity has been elegantly demonstrated in T98G glioblastoma cells, where introduction of a kinase-dead, dominant-negative mutant $C D K 9$ results in a gene expression profile that is distinct from that induced by flavopiridol [26]. While the latter study emphasizes the polypharmacology of flavopiridol, identifying precise targets associated with clinical toxicity has been challenging [27]. In addition, the pharmacokinetic properties of flavopiridol and other inhibitors such as dinaciclib require intravenous dosing, with different infusion schedules being explored in specific trials $[4,5,7,28-30]$. We therefore sought to develop smallmolecule inhibitors of CDK9 with an improved selectivity profile over other CDKs to more precisely drive MCL-1dependent tumor apoptosis and enhance the activity of the BCL-2 selective inhibitor venetoclax in hematologic malignancies. An additional goal of this program was to generate compounds with oral activity to enable the option of an all oral regimen for treating BCL-2, MCL-1 co-dependent tumors.

\section{Materials and methods}

\section{Reagents, cell culture, and treatment}

H929, MV4-11, HEL, U-937, KASUMI-1, KG-1, THP-1, SU-DHL-4, and A-431 cells were obtained from the American Type Culture Collection (ATCC), and SET-2, SKM-1, SHI-1, and NOMO-1 from Deutsche Sammlung von Mikroorganismen und Zellkulturen (DSMZ) and were cultured in the recommended media containing $10 \%$ fetal bovine serum (FBS) and $10 \mathrm{mM}$ L-glutamine (all from Invitrogen Corporation, Carlsbad, CA, USA). All cell lines were tested for authenticity by short tandem repeat profiling and mycoplasm by the AbbVie Core Cell Line Facility. TP53 status was determined by next-generation sequencing. SC-1 and OCI-Ly1 cell lines with acquired resistance to venetoclax (SC-1 199R and OCI-Ly1 199R, respectively) were generated and cultured as described by Tahir et al. [18]. Characterization of the $\mathrm{E} \mu-M y c$ lymphoma cell lines

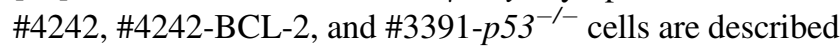
elsewhere [23, 31]. 
For in vitro experiments, venetoclax, A-1210477, A-1467729, and A-1592668 were dissolved in anhydrous DMSO. For in vivo experiments, A-1592668 was formulated in $2 \%$ DMSO $+5 \%$ Tween $80+20 \%$ [polyethylene glycol (PEG) $400+73 \%$ HPMC (2\% hydroxypropyl methyl cellulose in water) (Sigma, MO, USA) and venetoclax was formulated in $10 \%$ ethanol $+60 \%$ Phosal 50 PG (Sigma, MO, USA $+30 \%$ PEG 400 .

\section{CDK enzymatic and binding assays}

CDK enzyme activities were measured using LANCE ULight TR-FRET kinase assay reagents (PerkinElmer Inc. Waltham, MA, USA) and the signal generated using a LANCE Ultra Europium anti-phospho-MBP antibody (PerkinElmer Inc.) was evaluated using a PerkinElmer Envision in TR-FRET mode (excitation at $320 \mathrm{~nm}$ and emission at $615 / 665 \mathrm{~nm}$ ). In addition, compound affinity for CDK8 was determined using a TR-FRET binding assay and the resulting signal assessed on the PerkinElmer Envision using Lantha Screen settings: excitation 340, emission 495/520 nm.

\section{Cell viability}

Cells $\left(0.1 \times 10^{6} / \mathrm{ml}\right)$ were treated in $96-$ well plates for up to $24 \mathrm{~h}$ and cell viability determined by CellTiterGlo as described by the manufacturer's instructions (Promega Corporation, Madison, WI, USA). Responses were determined as a percentage of the control treated cells and $I C_{50} \mathrm{~S}$ determined from sigmoidal dose-response curves using GraphPad Prism (GraphPad Software, La Jolla, CA USA).

\section{Annexin-V /7-AAD staining}

Exponentially growing human tumor cell lines and the E $\mu$-Myc lymphoma cell lines \#4242, \#4242-BCL-2, and \#3391-p53 ${ }^{-/}[23,31]$ were treated with A-1592668 and/or venetoclax for the indicated times and the proportion of apoptotic cells was determined by flow cytometric assessment of annexin-V staining and PI uptake.

\section{Western blot analysis}

After treatment, cells were washed twice with ice-cold PBS containing $10 \% \mathrm{FBS}$, centrifuged at 1000 r.p.m. for $5 \mathrm{~min}$, and lysed in $50 \mu$ l of ice-cold Cell Lytic ${ }^{\mathrm{TM}}$ (Sigma) supplemented with protease (Roche Diagnostics Corporation, Indianapolis, IN, USA) and phosphatase (Sigma) inhibitors. Protein concentrations were determined by the BSA assay (Invitrogen Corporation) and $50 \mu \mathrm{g}$ of protein was electrophoresed by SDS-PAGE (Invitrogen Corporation) and separated proteins were transferred to nitrocellulose membranes. Blots were probed with anti-t-RNA pol-II (Covance,
Princeton NJ, USA; Cat \# MMS126R), anti-p-RNA pol-II (Bethyl Montgomery TX, USA; cat \# A300-654A), antiMCL-1 (Santa Cruz Biotechnology, La Jolla, CA, USA; Cat \# SC-12756), anti-PARP (BD Bioscience, CA, USA; Cat \# 556362), anti-caspase-3 (Cell Signaling Technology, Danvers, MA, USA; Cat \# 9662), anti-GAPDH (Cell Signaling Technology; Cat \# 2118), tubulin (Santa Cruz Biotechnology; Cat \# SC-8035) or $\beta$-actin (Sigma; Cat \# A2228) the indicated primary antibodies and detected using IRDye 680/ 800CW-conjugated secondary antibodies (LICOR Biosciences, NE, USA). Proteins were visualized utilizing the Odyssey ${ }^{\circledast}$ infrared imaging system (LI-COR Biosciences) and were not further manipulated with imaging software.

\section{In-cell western to determine RNA polymerase II phosphorylation (Ser2)}

A-431 cells were seeded in 96-well black plates at 10,000 cells/well overnight and then treated for $4 \mathrm{~h}$ with A-1592668 or A-1467729. Cells were subsequently fixed with $4 \%$ paraformaldehyde prior to incubation with either rabbit anti-p-RNA pol-II (Ser2; Bethyl Laboratories; Cat \# A300-654A) or rabbit IgG (Sigma; Cat \# I5006) overnight at $4{ }^{\circ} \mathrm{C}$. Cells were then washed with Delfia/Autodelfia wash buffer and incubated in the dark for $1 \mathrm{~h}$ with Alexa Fluor 488 goat anti-rabbit antibody and Hoechst stain. Expression of p-RNA poll-II (Ser2) was subsequently assessed on a Thermo Fisher CellInsight and $I C_{50}$ values were determined by nonlinear regression analysis of the resulting concentration response data.

\section{Isolation of primary lymphoma cells and ex vivo treatment}

Primary lymphoma cells were isolated from tissue biopsies according to protocols approved by the Institutional Review Board of the Cleveland Clinic and cultured in RPMI-1640 media containing 20\% FBS prior to treatment with A-1467729, venetoclax, or their combinations for $6 \mathrm{~h}$. Cell death was subsequently determined from the percentage 7-AAD (BD Bioscience) positive lymphoma population by flow cytometry (BD FACScalibur ${ }^{\mathrm{TM}}$, BD Bioscience, Franklin Lakes, NJ, USA). Alternatively, samples were processed for assessment of t-RNA pol-II \& p-RNA pol-II expression by western blot analysis as described or BCL-2, MCL-1, $\beta$-actin, and PARP (all Cell Signaling Technology; Cat \# 4223, 5453, 3700, and 9542 respectively).

\section{Mouse models of lymphoma and leukemia}

Subcutaneous tumor cell line-derived xenograft (CDX), AML patient derived xenograft (PDX), and $\mathrm{E} \mu-\mathrm{Myc}$ lymphoma studies were conducted in accordance with 
guidelines approved by the Institutional Animal Care and Use Committees at AbbVie Inc., the University of Texas MD Anderson Cancer Center, and Peter MacCallum Cancer Centre, respectively. Specific experimental details are included in the supplementary methods and related figure legends.

\section{Statistical analysis}

Data are represented as the mean \pm s.e.m. In all cases, $n$ refers to the number of independent experiments. The Bliss independence model was used to evaluate synergy in vitro $[32,33]$. The significance between the rankings of two variables was determined by Spearman's rank correlation co-efficient and the Mantel-Cox test used to assess significance between Kaplan-Meier survival curves, both using GraphPad Prism (GraphPad Software).

\section{Results}

\section{Discovery and characterization of the CDK9- selective inhibitors A-1467729 and A-1592668}

A-1467729 (Fig. 1) was identified through SAR studies as a potent CDK9 inhibitor $\left(I C_{50}=1.2 \mathrm{nM}\right)$ with $>1000 \times$ selectivity over CDK1 and CDK2, as well as the transcriptional kinases CDK7 and CDK8. The binding of A-1467729 to CDK4 and CDK6 was greater in comparison, affording selectivity of $\sim 12$ - and 240-fold, respectively. At the cellular level, A-1467729 inhibited phosphorylation of RNA pol-II (p-RNA pol-II; Ser2) with a potency of

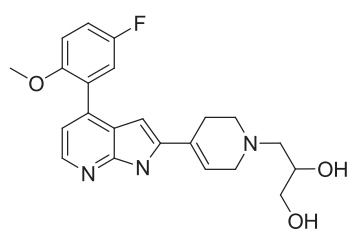

A-1467729

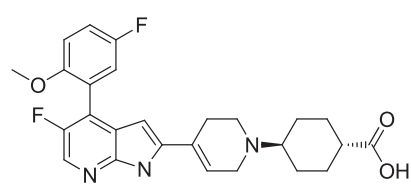

A-1592668
Fig. 1 Chemical structures of A-1467729 and A-1592668.
$26.7 \mathrm{nM}$. This pharmacodynamic effect translated to potent cell killing in the MCL-1 dependent hematologic tumor cell lines $[34,35] \mathrm{H} 929\left(I C_{50}=39 \mathrm{nM}\right)$ and MV4-11 $\left(I C_{50}=\right.$ $42.4 \mathrm{nM}$ ) following $24 \mathrm{~h}$ of incubation (Table 1). Pharmacokinetic studies in rats $(1 \mathrm{mg} / \mathrm{kg})$ demonstrated a Clp of $2.1 \mathrm{~L} / \mathrm{h} / \mathrm{kg}$ upon intravenous dosing; while this compound showed several attributes of a productive tool compound for in vitro studies, decreased clearance was prioritized in order to generate a suitable tool for in vivo studies as well as to provide a productive starting point for an oral drug discovery program (Table 2). To this end additional SAR studies were performed with the goal of decreasing clearance to $<20 \%$ liver blood flow while maintaining the affinity towards CDK9. Modifications primarily to the righthand portion of the pharmacophore afforded A-1592668 (Fig. 1). The incorporation of the acid moiety in A-1592668 conferred decreased clearance $(\mathrm{Clp}=0.63)$ upon IV administration in rat. Similar levels of oral absorption and bioavailability were observed for both compounds upon oral gavage (Table 2). A-1592668 retained potency against CDK9 $\left(I C_{50}=2.6 \mathrm{nM}\right)$ as well as $>500 \times$ selectivity over transcriptional kinases CDK7 and CDK8. While affinity for CDKs 1 and 2 were increased relative to predecessor A-1467729, >100-fold selectivity was maintained, with the overall affinity to CDKs 4 and 6 being similar. Finally, A-1592668 demonstrated robust pharmacodynamic marker movement and cell killing in MCL-1 dependent cell lines (Table 1), yet spared non-MCL-1 dependent cell lines (Supplementary Table 1; [34]), thus phenocopying the in vitro effects of A-1467729 and indicating that cell killing in H929 and MV4-11 cells was driven via inhibition of CDK9. A-1592668 was thus selected as the primary tool to assess the effects CDK9 inhibition in vivo.

\section{A-1592668 induces mechanism-based apoptosis in tumor cells dependent upon MCL-1 for survival}

Treatment of H929 or MV4-11 cells with A-1592668 induced a dose- and time-dependent loss in MCL-1 expression that correlated with a decrease in p-RNA pol-II (Ser2; Fig. 2a). The observed rapid loss in MCL-1

Table 1 Biochemical characterization of A-1467729 and A-1592668.

\begin{tabular}{|c|c|c|c|c|c|c|c|c|c|c|}
\hline & \multicolumn{7}{|c|}{$\mathrm{CDK} \mathrm{IC}_{50}(\mu \mathrm{M})$} & \multirow{2}{*}{$\begin{array}{c}\mathrm{ICW} \mathrm{IC}_{50}(\mu \mathrm{M}) \\
\mathrm{A} 431\end{array}$} & \multicolumn{2}{|c|}{ Cell viability $\mathrm{IC}_{50}(\mu \mathrm{M})$} \\
\hline & CDK1 & CDK2 & CDK4 & CDK6 & CDK7 & CDK8 & CDK9 & & H929 & MV4-11 \\
\hline A-1467729 & 3.04 & 1.36 & 0.0155 & 0.298 & $>20$ & 3.11 & 0.0012 & 0.0267 & 0.039 & 0.0424 \\
\hline A-1592668 & 0.348 & 0.328 & 0.0162 & 0.101 & 14.4 & 1.61 & 0.0026 & 0.106 & 0.040 & 0.138 \\
\hline
\end{tabular}

The CDK binding and enzymatic activity of A-1467729 and A-1592668 were determined as described in the materials and methods, and $I C_{50} \mathrm{~s}$ calculated. A-431 cells were treated with A-1467729 $(n=1)$ or A-1592668 $(n=2)$ for $4 \mathrm{~h}$ and the impact on RNA pol-II phosphorylation (Ser2) determined by in-cell western (ICW) and $I C_{50}$ s calculated. H929 and MV4-11 cells were treated with A-1467729 or A-1592668 for $24 \mathrm{~h}$ and the effect on cell viability determined using CellTiterGlo. $I C_{50}$ s were calculated from the resulting dose response curves. Data from CDK binding and enzymatic assays, and viability assays are presented as the mean of at least three independent experiments 
expression was accompanied by the induction of apoptosis as demonstrated by the processing of caspase- 3 and PARP cleavage by western blot analysis ( $\leq 8 \mathrm{~h}$; Fig. $2 \mathrm{a}, \mathrm{b})$, and the externalization of phosphatidylserine (Fig. 2c, d). A-1592668-mediated apoptosis of both H929 and MV4-11

Table 2 Pharmacokinetic properties of A-1467729 and A-1592668.

\begin{tabular}{lllll}
\hline & \multicolumn{4}{c}{ Selected rat PK parameters } \\
\cline { 2 - 5 } & $1 \mathrm{mg} / \mathrm{kg} \mathrm{IV}$ & \multicolumn{3}{c}{$\mathrm{mg} / \mathrm{kg}$ PO } \\
\hline & $\mathrm{CL}_{\mathrm{p}}(\mathrm{L} / \mathrm{h} / \mathrm{kg})$ & $V_{\mathrm{ss}}(\mathrm{L} / \mathrm{kg})$ & $\mathrm{AUC}(\mathrm{ng} * \mathrm{~h} / \mathrm{ml})$ & $F(\%)$ \\
$\mathrm{A}-1467729$ & 2.1 & 19.6 & 181.3 & 37 \\
$\mathrm{~A}-1592668$ & 0.63 & 2.7 & 452 & 28 \\
\hline
\end{tabular}

Rats $(n=3)$ were dosed with A-1467729 and A-1592668 at $1 \mathrm{mg} / \mathrm{kg}$ IV or PO and clearance $\left(\mathrm{CL}_{\mathrm{p}}\right)$, volume of distribution at steady state $\left(V_{\mathrm{ss}}\right)$, plasma concentration (area under the curve; AUC), and bioavailability (\%F) determined (See Supplementary Materials and Methods) cells was inhibited by pre-treatment with the pan-caspaseinhibitor Q-VD-oph (Fig. 2c, d). A-1592668 induced regression of MV4-11 tumors in vivo that was maintained for up to 35 days post-treatment without dramatically impacting weight loss (Fig. 2e). To further assess the antitumorigenic activity of A-1529668 in MCL-1 dependent hematologic malignancies, murine \#4242 E $\mu$-myc lymphoma cells [23] were treated in vitro with A-1592668. A-1592668 treatment resulted in a dose-dependent increase in Annexin-V/PI staining (Fig. 3a) that was associated with a decrease in Mcl-1 and p-RNA pol-II expression (Fig. 3b). B-cell lymphomas deficient in p53, \#3391 [31], were as sensitive to A-1592668 in vitro as their wild-type counterparts (Supplementary Fig. 1). Transplant of \#4242 tumors into immunocompetent mice resulted in the aggressive development of lymphoma [23] and death within 14 days post-transplant, with a median survival of 13.5 days.

\section{a}

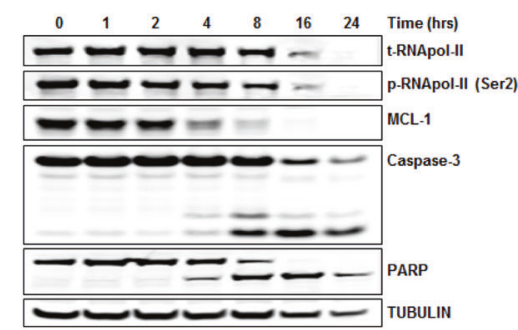

H929, 6 hrs treatment

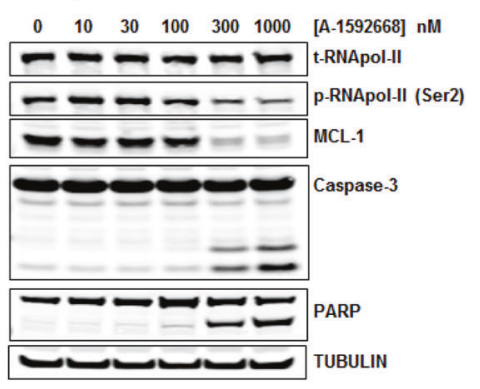

e

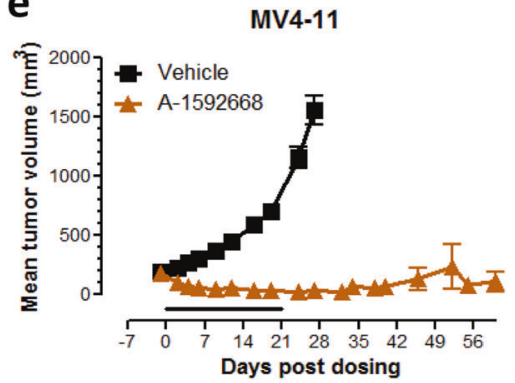

b

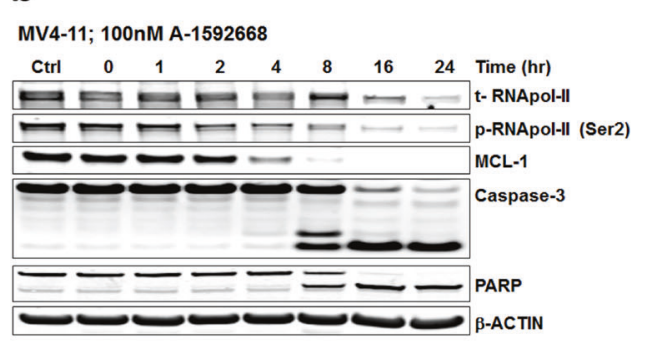

MV4-11, 6 hrs treatment

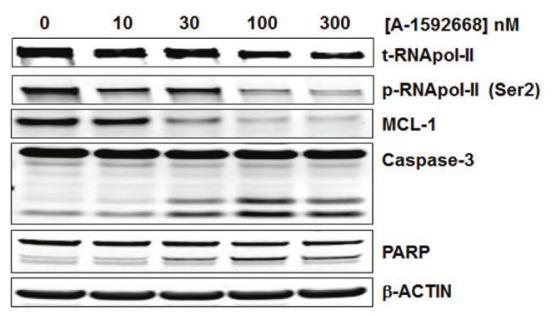

C

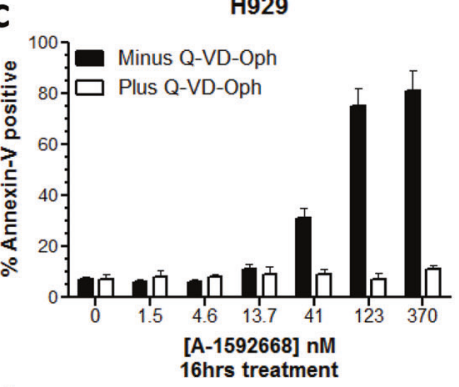

d

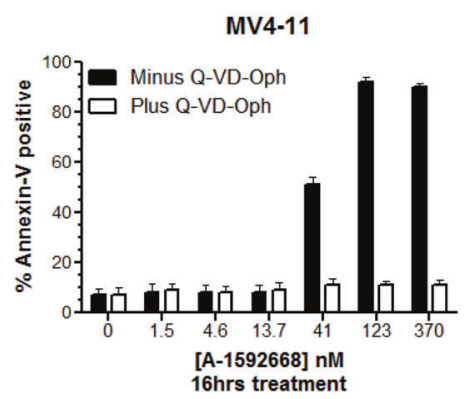

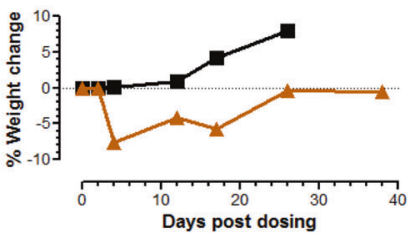

Fig. 2 A-1592668 induces on-target cell death in MCL-1 dependent tumor cell lines. H929 or MV4-11 cells were treated in vitro with A-1592668 at the indicated concentrations and times, and the impact on total and phosphorylated RNA polymerase-II (t-RNA pol-II and p-RNA pol-II respectively), MCL-1, Caspase-3, and PARP determined by western blot utilizing tubulin or $\beta$-actin as a loading control $(\mathbf{a}, \mathbf{b})$. Alternatively, MV4-11 or H929 cells were treated with A-1592668 for
$16 \mathrm{~h}$ in the presence or absence of the pan-caspase inhibitor Q-VDOph $(100 \mu \mathrm{M})$ and the percentage Annexin-V-positive cells determined by flow cytometry $(\mathbf{c}, \mathbf{d})$. Mice bearing MV4-11 tumors were treated with A-1592668 (5 mg/kg PO, twice a week for 3 weeks or the vehicle ( $n=6$ mice per group), and the effect on tumor growth determined as described in the materials and methods. The impact on weight was also assessed (e). 
a

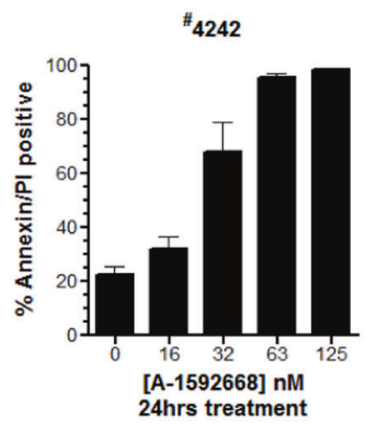

b

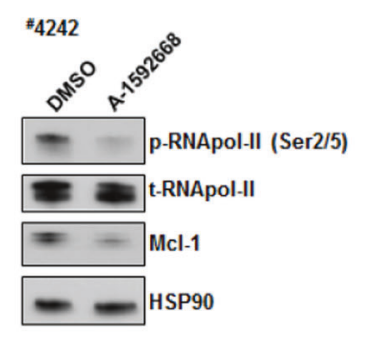

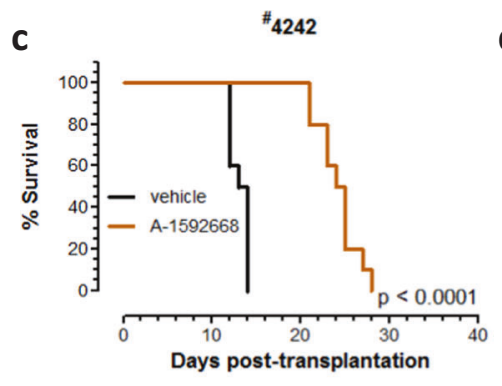

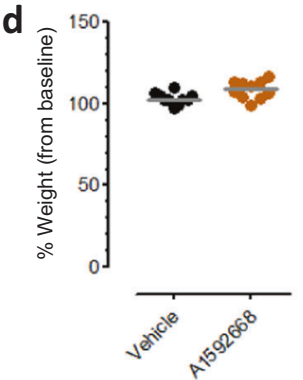

e
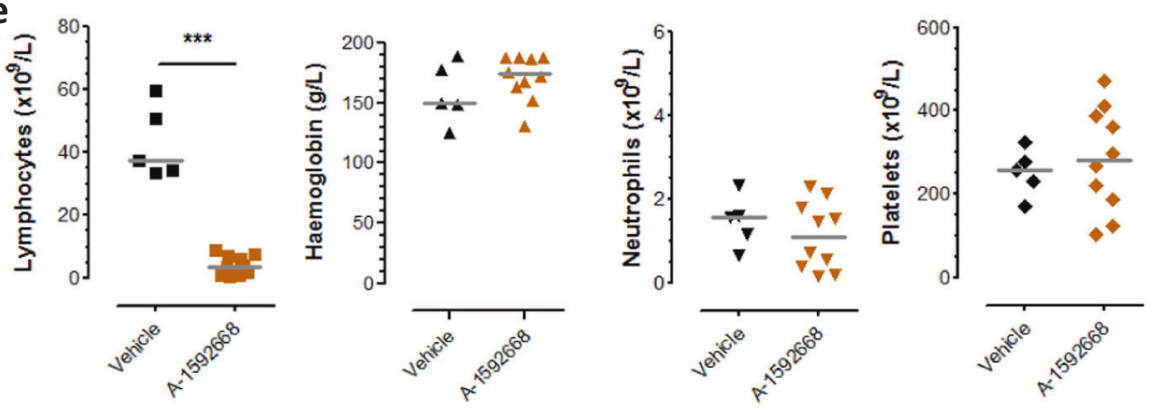

f

g

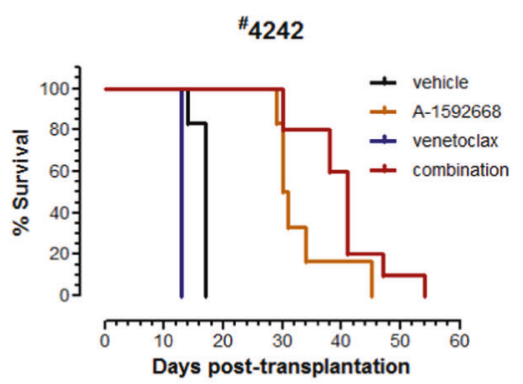

h

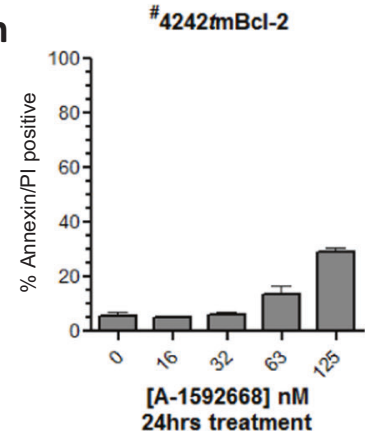

Fig. 3 A-1592668 is active in an E $\mu$-myc mouse model of lymphoma. E $\mu$-myc mouse lymphoma cells (\#4242) were treated with A-1592668 in vitro for $24 \mathrm{~h}$ and the percentage Annexin-V/PI-positive cells determined by flow cytometry. Data are presented as the mean \pm s.e.m. of three independent experiments (a). Alternatively, E $\mu$-myc mouse lymphoma cells (\#4242) were treated with A-1592668 (100 nM) in vitro for $3 \mathrm{~h}$ and the effects on Mcl-1 and p-RNA pol-II (Ser2/5) determined by western blot utilizing t-RNA pol-II and HSP90 as loading controls (b). C57B1/6 mice bearing \#4242 tumors were treated with A-1592668 (3 mg/kg PO, three times per week for two weeks, $n=10)$ or vehicle $(n=10)$ and the impact on animal survival (c), body weight (d), lymphocytes, hemoglobin, neutrophils, and platelets determined (e). Statistical difference was determined using an unpaired

Treatment of mice harboring \#4242 tumors with A-1592668 resulted in a significant increase in survival (median survival of 24.5 days, $p<0.0001)$ over the vehicle-treated control mice (median survival of 13.5 days; Fig. 3c) with no significant weight loss (Fig. 3d) and was associated with a decrease in lymphocyte burden without impacting hemoglobin, neutrophil, or platelet counts (Fig. 3e). Activity of A-1592668 in vivo was deemed on-target as evident by a decrease in tumor Mcl-1 expression and a loss in RNA pol-II phosphorylation (Ser 2/5; Fig. 3f). In contrast to $t$-test, where $p<0.001(* * *)$ was considered significant. In a separate cohort, untreated mice were allowed to establish bulky lymph nodal disease for 12 days before treatment with vehicle or a single dose of A-1592668 (3 mg/kg PO) for $3 \mathrm{~h}$ before tumors were excised and the impact on p-RNA pol-II (Ser2/5) and Mcl-1 determined by western blot using HSP90 as a loading control (f). C57B1/6 mice bearing \#4242 tumors were treated as in $\mathbf{c}$ in the presence or absence of venetoclax $(50 \mathrm{mg} / \mathrm{kg}$ PO, every day for two weeks). Median survival rates for each treatment group are presented in Supplementary Table 2a $(\mathbf{g})$. E $\mu$-myc mouse lymphoma cells overexpressing BCL-2 (\#4242tmBcl2) were treated with A-1592668 in vitro for $24 \mathrm{~h}$ and the percentage Annexin-V/PI positive cells determined by flow cytometry. Data are presented as the mean \pm s.e.m of three independent experiments (h).

A-1592668 which induced robust apoptosis at low-nM concentrations (Fig. 3a), the BCL-2 selective inhibitor venetoclax (ABT-199) was substantially less active in \#4242 cells in vitro (Supplementary Fig. 2) and did not provide any survival benefit in vivo when compared with vehicle-treated animals (Fig. 3g). However, co-treatment of \#4242 tumors in vivo with A-1592668 and venetoclax provided an additional survival advantage over A-1592668 treatment alone, extending median survival from 30.5 days to 41 days (Fig. 3g and, Supplementary Table 2a). 


\section{BCL-2 inhibition sensitizes DLBCL cells to CDK9- selective inhibitors}

MCL-1 expression has been identified as an important resistance factor limiting the activity of venetoclax in a number of hematologic tumor types [36] including lymphoma [17-19]. Reciprocally, overexpression of $B c l 2$ in E $\mu$-myc tumors inhibited A-1592668 activity in vitro (Fig. 3h). Because loss in p-RNA pol-II and MCL-1 expression occurs rapidly after CDK9 inhibition ( $\leq 8 \mathrm{~h}$; Fig. 2a, b), we hypothesized that concomitant BCL-2 inhibition would quickly induce strong synergistic apoptosis within this time frame in cell lines with either inherent (SU-DHL-4) or acquired resistance to venetoclax (OCI-Ly1 199R and SC-1 199R; [18]). As expected,
A-1592668 synergized with venetoclax in killing SU-DHL-4, OCI-Ly1 199R, and SC-1 199R cells (Fig. 4a, b), and this combination resulted in greater tumor growth inhibition over either agent alone in a SU-DHL-4 xenograft model (Fig. 4c; venetoclax alone versus A-1592668 alone vs combination, $55 \%$ TGI versus $72 \%$ TGI vs $91 \%$ TGI respectively). To further explore the effect of co-inhibiting CDK9 and BCL2 , we treated a panel of primary diffuse large B-cell lymphoma (DLBCL) and follicular lymphoma (FL) patient samples ex vivo with this combination. CDK9 inhibition with A-1467729 resulted in a loss in p-RNA pol-II (Ser2) that aligned with a loss in MCL-1 expression (Fig. 4d) and enhanced the cell killing activity of venetoclax (Fig. 4e) in several primary DLBCL and FL patient samples ex vivo.
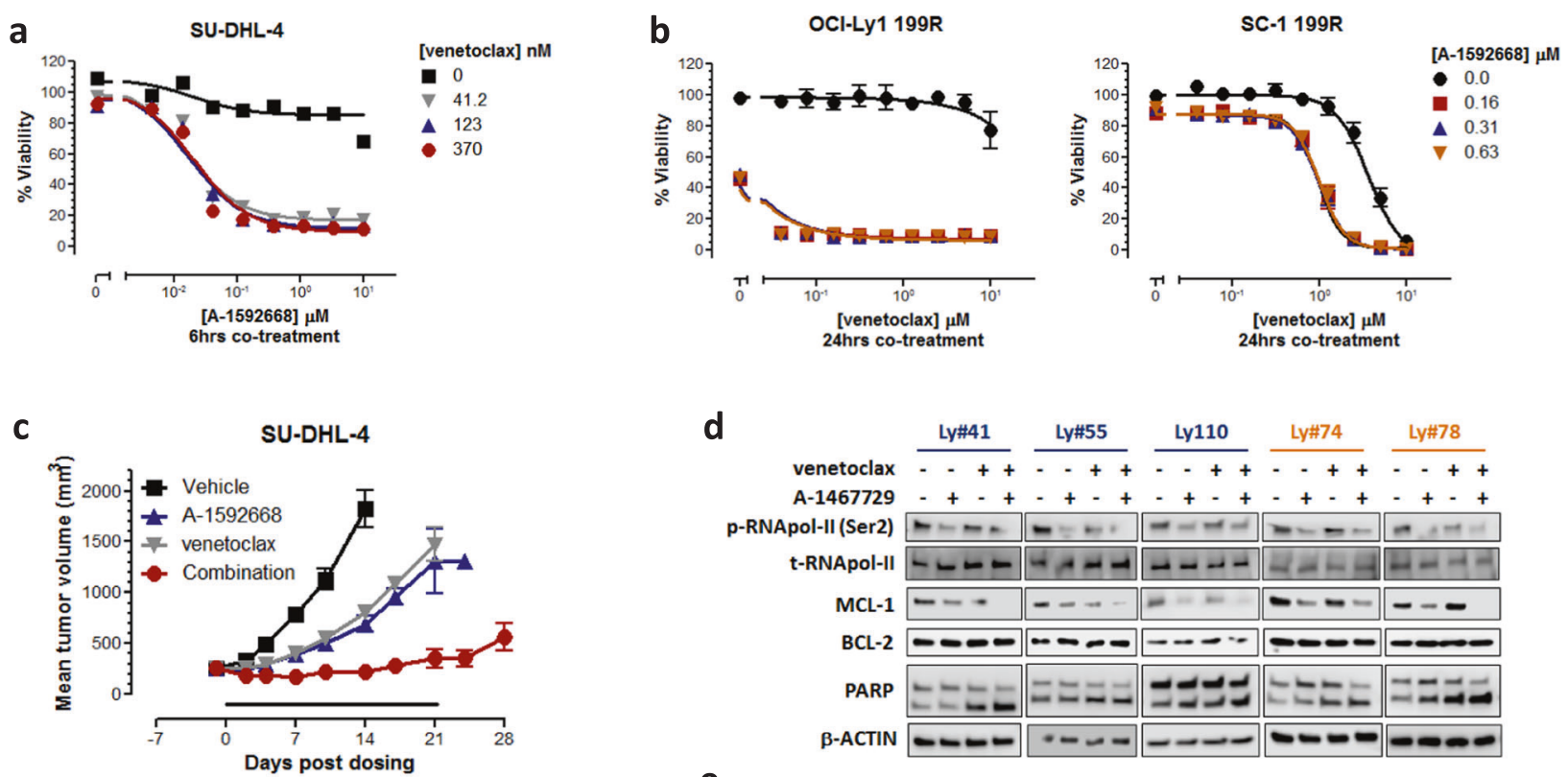

d
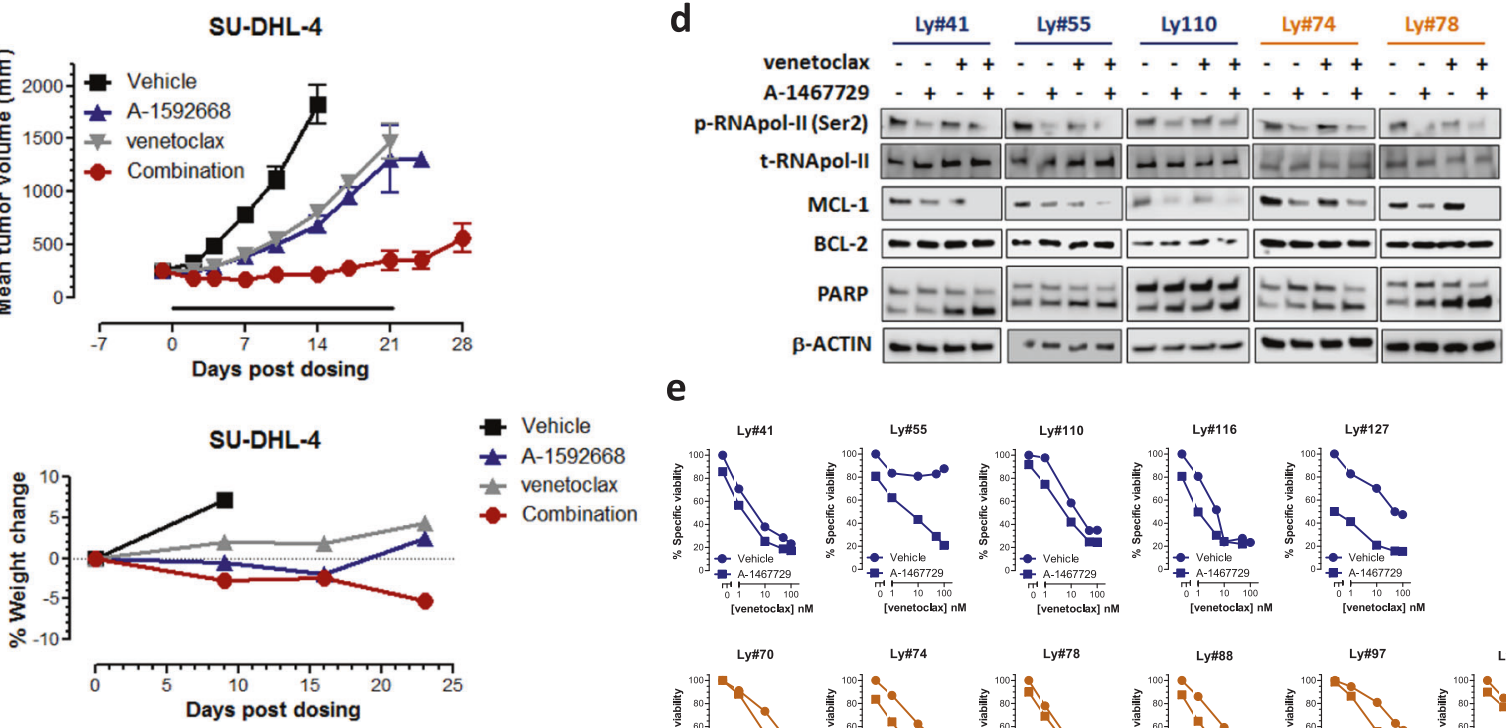

e
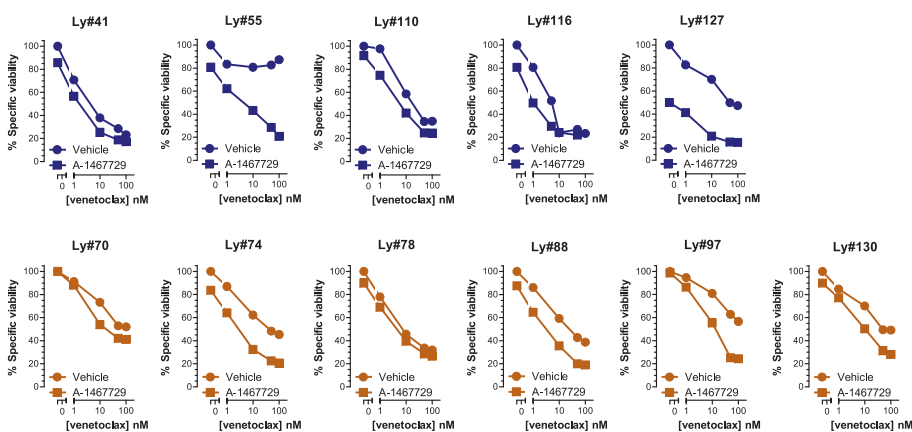

Fig. 4 A-1592668 sensitizes NHL cells to venetoclax. SU-DHL-4 cells $(\mathbf{a} ; n=1)$, OCI-LY1 199R, or SC-1 199R (b) were treated with A-1592668 in combination with venetoclax in vitro for the indicated times and the impact on cell viability determined by CellTiterGlo. Data in $\mathbf{b}$ are presented as the mean \pm s.e.m of three independent experiments. A-1592668 given orally at $1.5 \mathrm{mg} / \mathrm{kg}$ three times a week for 3 weeks either alone or in combination with venetoclax given orally at $50 \mathrm{mg} / \mathrm{kg}$ once a day for 3 weeks in the SU-DHL-4 flank xenograft model ( $n=9$ mice per treatment group). Data are presented as the mean tumor volume \pm s.e.m. The impact on mouse weight is also shown (c). Primary DLBCL (blue) and FL (orange) patient samples were treated ex vivo with venetoclax in the presence or absence of A-1467729 (10 nM) for $6 \mathrm{~h}$ and the impact on total and phosphorylated RNA polymerase-II (t-RNA pol-II and p-RNA pol-II respectively), MCL-1, BCL-2, PARP cleavage, and $\beta$-actin determined by western blot (d), or the effect on cell viability determined using 7-AAD uptake (e). 
The orally bioavailable CDK9-selective inhibitor A-1592668 enhances the efficacy of venetoclax in preclinical models of AML

AML represents a diverse disease that is typified by high degrees of heterogeneity [37]. This manifests in different phenotypic responses of AML cell lines to inhibitors of the antiapoptotic BCL-2 family [34]. Although clinical activity with venetoclax has been demonstrated in a phase-II trial in heavily pre-treated R/R AML patients [38], more robust responses have been observed in the combination setting [39]. Therefore, we evaluated venetoclax activity in combination with the CDK9 inhibitor A-1592668 or the MCL-1 inhibitor
A-1210477 in a panel of AML cell lines. A-1210477 and A-1592668 were each broadly synergistic with venetoclax in killing a number of AML cell lines in vitro after $6 \mathrm{~h}$ of treatment (Fig. 5a, b; Supplementary Fig. 3). Representative data are shown for SKM-1, where venetoclax, A-1210477, or A-1592668 each lacked substantial single agent activity at this early time point, yet strong activity was observed when venetoclax was combined with either agent (Fig. 5a, b; Supplementary Fig. 3a, b). Although A-1592668 treatment alone reduced p-RNA pol-II (Ser 2) and MCL-1 expression in SKM-1 cells in a dose- and time-dependent manner, robust caspase-3 activation and PARP cleavage (indicators of apoptosis induction) were not observed without the presence
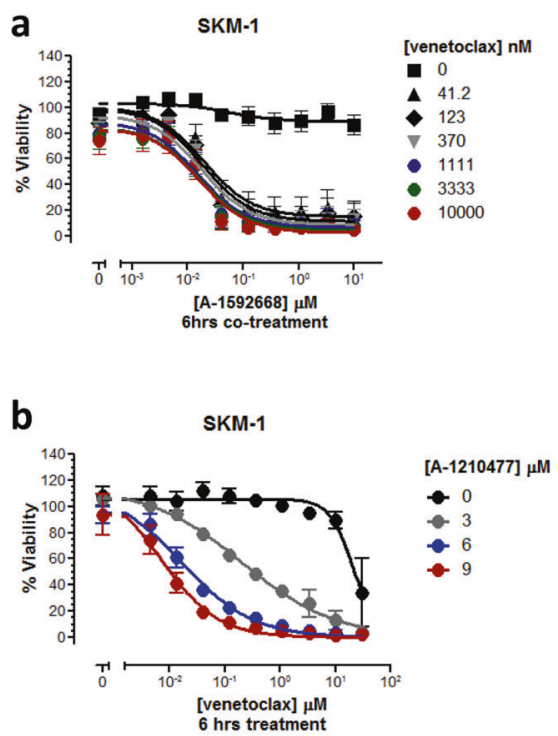

d

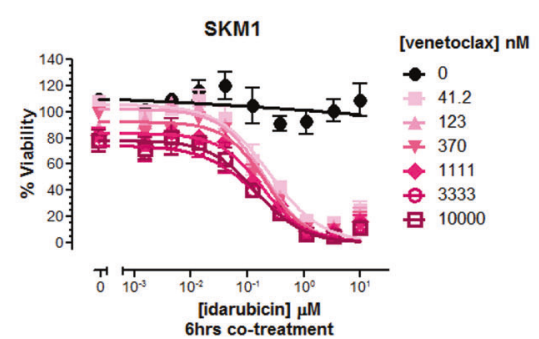

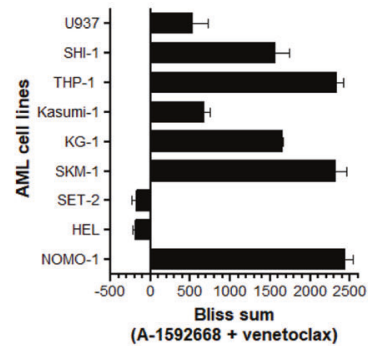
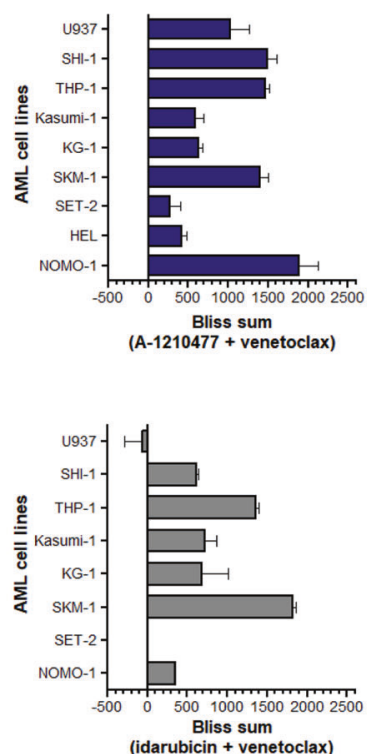

C
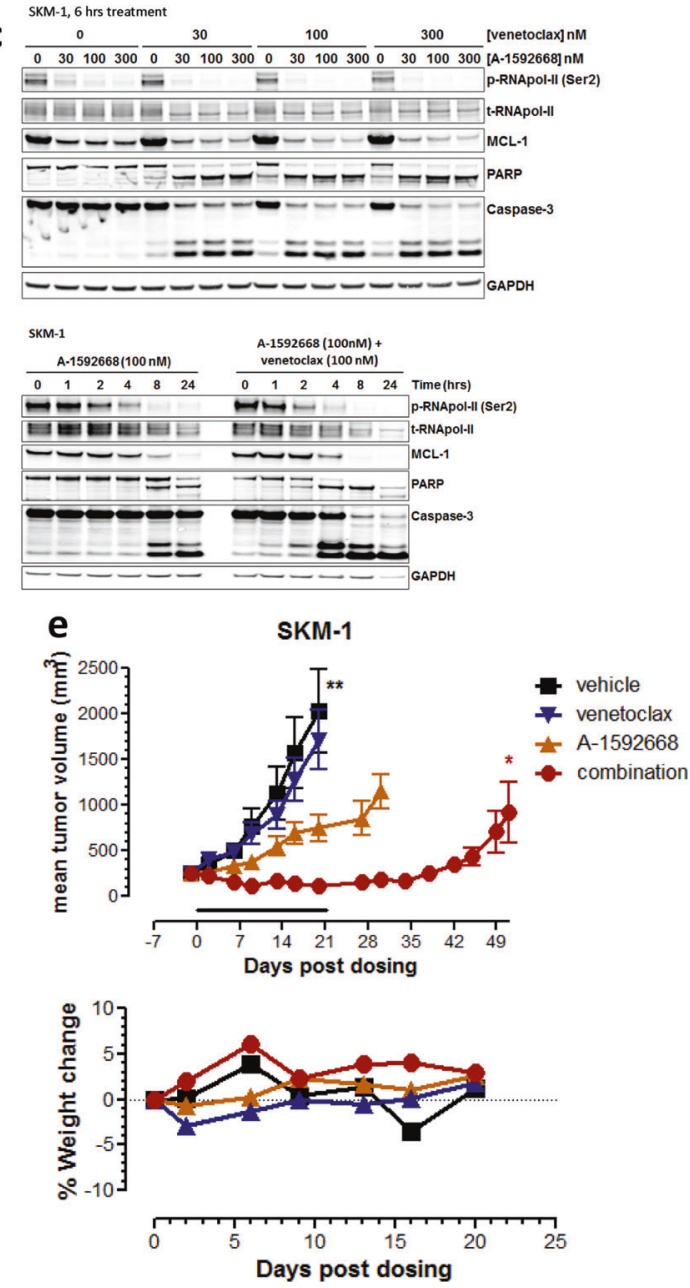

Fig. 5 A-1592668 sensitizes AML cells to venetoclax. A panel of AML cell lines was treated with A-1592668 (a; $n=3$ independent experiments for each cell line), A-1210477 (b; $n=3$ independent experiments for each cell line) or idarubicin (d; $n=2$ independent experiments for each cell line) in combination with venetoclax for $6 \mathrm{~h}$ in vitro and the effect on cell viability determined by CellTiterGlo. Dose-response curves in the SKM-1 AML tumor cell line is shown, and the synergy assessed using the Bliss independence model, where Bliss Sums represent the cumulative Bliss scores across the combination matrix. Data are presented as the mean \pm s.e.m. The impact of A- 1592668 treatment in the presence or absence of venetoclax on total and phosphorylated RNA polymerase-II (t-RNA pol-II and p-RNA pol-II respectively), MCL-1, Caspase-3, and PARP determined by western blot utilizing GAPDH as a loading control (c). The SKM-1 flank xenograft model was given A- 1592668 orally at $2.5 \mathrm{mg} / \mathrm{kg}$ twice a week for 3 weeks either alone or in combination with venetoclax orally at $50 \mathrm{mg} / \mathrm{kg}$ once a day for 3 weeks. Data are presented as the mean tumor volume \pm s.e.m. of eight mice per treatment arm. The impact on mouse weight is also shown. $* * 2 \times$ found dead and excluded from analysis on day $3, * 1 \times$ moribund and excluded from analysis on day $6(\mathbf{e})$. 
of venetoclax (Fig. 5c). Interestingly, the BCL- $\mathrm{X}_{\mathrm{L}}$ dependency of SET-2 and HEL cells [34] could be overcome by the A-1592668/venetoclax combination when employed for a longer treatment period ( 24 h; Supplementary Figs. 3 and 4). Further, potency and synergy between venetoclax and A-1592668 were greater than that obtained with venetoclax in combination with idarubicin (Fig. 5d and Supplementary Fig. 3c), a treatment option in AML reported to downregulate MCL-1 expression [36]. CDK9i/venetoclax synergy strongly correlated with A-1210477/venetoclax synergy, but not idarubicin/venetoclax synergy (Supplementary Fig. 5) indicating that additional factors beyond MCL-1 may contribute to the mechanism of idarubicin's combination activity with venetoclax (Fig. 5d). The strong combinational activity observed in vitro with A-1592668 and venetoclax was reflected in a xenograft model of AML. Co-treatment of mice bearing SKM-1 tumors with A-1592668 and venetoclax induced tumor regression and substantial inhibition of tumor growth (94\% TGI) that was superior to either agent as a monotherapy (venetoclax, 16\% TGI; A-1592668, 63\% TGI) without impacting weight loss (Fig. 5e). The CDK9 inhibitor/venetoclax combination also improved the duration of response (320\%) when compared with that obtained with venetoclax alone (11\%) and A-1592668 alone (100\%).

To further interrogate the potential impact of CDK9 inhibition on venetoclax activity in AML, we treated two AML PDX models including one possessing the fms-like tyrosine kinase 3-internal tandem duplication (FLT3-ITD) mutation (PDX 4095636); a genomic aberration identified as a potential resistance factor to venetoclax in AML [40]. Engraftment rates across groups for each AML PDX model were equivalent prior to treatment (Supplementary Fig. 6). Although treatment of PDX 4095636 was initiated at a lower engraftment percentage than that in PDX 3899936 (Supplementary Fig. 6), median survival of the vehicle groups did not substantially differ (71 days versus 67.5 days respectively). Importantly, A-1592668/venetoclax co-treatment provided a significant survival advantage over either agent alone or the vehicle control group in both AML PDX models (Fig. 6a, c; Supplementary Table $2 \mathrm{~b}, \mathrm{c})$ that was associated with a decrease in the engraftment percentage (Fig. 6b, d).

\section{Discussion}

Herein we describe the discovery of a potent small-molecule inhibitor of CDK9 that is highly active in MCL-1 dependent AML (MV4-11) and MM (H929) cell lines, and a mouse lymphoma (\#4242) cell line in vitro. This activity is characterized by inducing mechanism-based apoptosis associated with a rapid loss in RNA pol-II phosphorylation and MCL-1 expression evident within $4 \mathrm{~h}$ of treatment. Oral administration of A-1592668 induced robust tumor regression in the MV4-11 xenograft model of AML and also provided an extension of median survival in an immunocompetent dependent mouse model of lymphoma [23, 31] with associated reduction of RNA pol-II phosphorylation and MCL-1 expression. No significant impact on weight loss, hemoglobin, neutrophil, or platelet counts were observed, indicating that A-1592668 is well-tolerated in preclinical models of human cancer.

Conditional deletion of $\mathrm{Mcll}$ in mouse models of AML [41] and myc-driven B-cell lymphoma [42], and the activity of selective small-molecule inhibitors of MCL-1 in human NHL, MM, and AML cell lines [34, 35, 43] emphasize the importance of inhibiting MCL-1 function for potential therapeutic benefit in hematologic malignancies. This notion is further underscored here with the use of selective CDK9 inhibitors that transcriptionally repress MCL-1 expression to indirectly inhibit its antiapoptotic function. Furthermore, transcriptional changes to other short-lived survival factors via CDK9 inhibition can augment antitumor effects of targeting MCL-1 [25]. Collectively our data are supportive of inhibiting CDK9 as a monotherapy in subsets of NHL and AML. However, previously published preclinical data also highlight the contributions of BCL-2 and BCL- $\mathrm{X}_{\mathrm{L}}$ in maintaining the survival of these malignancies $[34,17,19,36]$, which may limit the breadth of single agent CDK9 inhibitor activity. A-1592668 alone did not induce cell death in AML cell lines that do not depend upon MCL1 for survival [34] (Supplementary Table 1). Reciprocally, while the BCL-2 selective inhibitor venetoclax (ABT-199) is active as a monotherapy in AML patients (19\% ORR) [38] and NHL (44\% ORR) [44], the ORR is not of the same magnitude as those observed in CLL (ORR of 79\%) [16], indicating that additional survival factors maintain hematologic tumor cell survival beyond BCL-2. Co-expression of MCL-1 and BCL-2 has been described in primary DLBCL patient samples [19], with inherent and acquired resistance to venetoclax attributed to the aberrant expression and function of other BCL-2 family members [17-19, 36, 45]. Since $B c l 2$ overexpression in E $\mu$-myc tumors limited the activity of A-1592668 in vitro, we assessed the combinatorial activity of A-1592668 or the related analog A1467729 with venetoclax in preclinical models of NHL and AML. Significant synergy was observed in numerous NHL and AML cell lines in vitro, additionally enhancing the activity of venetoclax in DLBCL and FL patient samples ex vivo. Combinational activity between venetoclax and CDK9 inhibitors in vitro was rapid, evident within $8 \mathrm{~h}$ of co-treatment and aligning with the swift loss in MCL-1 protein expression following its transcriptional repression coupled with its short protein half-life [46]. Further, the A-1592668 plus venetoclax combination inhibited tumor growth to a greater extent than either agent alone in xenograft models of AML (SKM-1) and DLBCL (SU-DHL-4) 


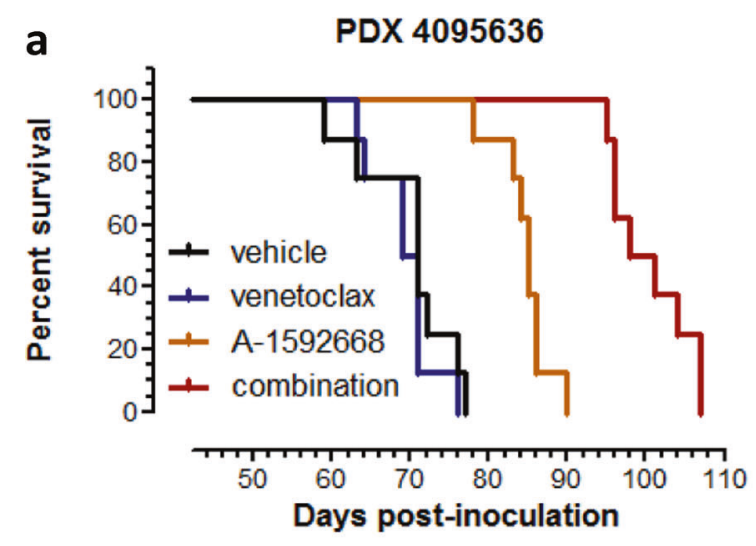

b

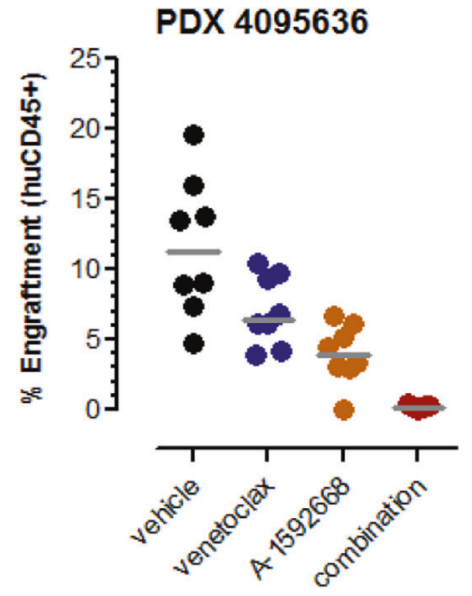

C

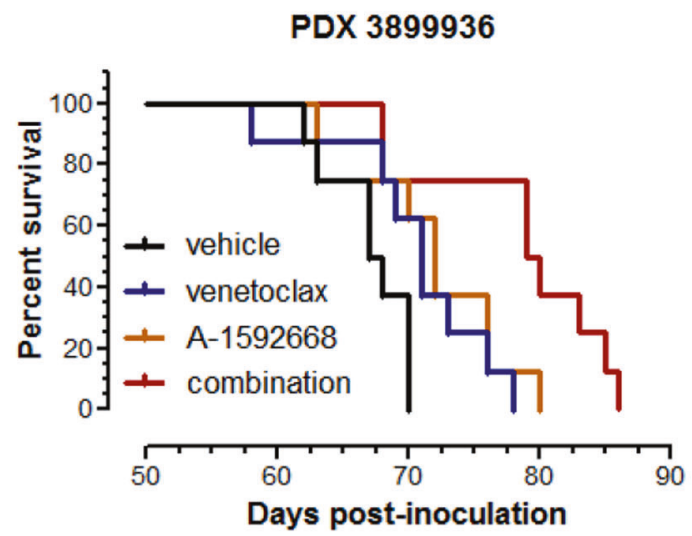

d

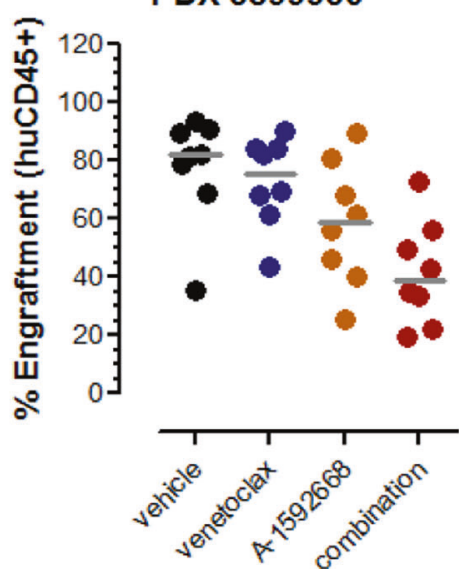

Fig. 6 A-1592668 is active in PDX models of AML with added survival benefit achieved in combination with venetoclax. NSG mice bearing AML-PDX derived cells (PDX 4095636 and PDX 3899936) were treated A- 1592668 orally at $4 \mathrm{mg} / \mathrm{kg}$ twice a week for 3 weeks either alone or in combination with venetoclax orally at $50 \mathrm{mg} / \mathrm{kg}$ once a day for 3 weeks and the impact on survival $(n=8$ per treatment group; a, c) and engraftment in the periphery one week after treatment (day $7 ; \mathbf{b}, \mathbf{d}$ ) determined. Levels of engraftment at the initiation of treatment are shown in Supplementary Fig. 6. A-1592668 provided a significant survival benefit over the vehicle treated mice in both PDX

and provided significant survival benefit in the $\mathrm{E} \mu-m y c$ tumor mouse model of lymphoma. Although venetoclax was active as a single agent in primary FL and DLBCL samples ex vivo, monotherapy did not induce " $100 \%$ kill", which may be attributed to the presence of MCL-1 protein. CDK9 inhibitor co-treatment reduced MCL-1 protein expression and enhanced venetoclax activity in many of the DLBCL and FL patient samples both in terms of the depth of response as well as $I C_{50}$. This indicates that although the $t$ $(14 ; 18)$ translocation is found in approximately $90 \%$ of FL patient samples [47], it does not necessarily confer BCL-2 survival dependency and may reflect the lower response rate observed in FL patients receiving venetoclax treatment (44\% ORR) [44] compared with those observed in CLL models (PDX 4095636, $p<0.0001$; PDX 3899936, $p=0.0214$ ) whereas venetoclax provided a significant survival benefit in PDX $3899936(p=0.0283)$ but not in PDX $4095636(p=0.3148)$. The combination of A-1592668 and venetoclax provided a significant survival benefit in PDX 4095636 over mice treated with vehicle or either agent alone $(p<0.0001)$. The combination of A-1592668 and venetoclax provided a significant survival benefit in PDX 3899936 over mice treated with vehicle $(p<0.0001)$, venetoclax alone $(p=$ $0.0065)$, or A-1592668 alone $(p=0.0307)$. Median survival rates for each treatment group are shown in Supplementary Table $2 b$, c.

patients (ORR of 79\%) [16]. Interestingly, targeting both BCL-2 and MCL-1 with venetoclax and CDK9 inhibitors respectively, was able to induce synergistic cell death in AML cell lines dependent upon BCL- $\mathrm{X}_{\mathrm{L}}$ for survival (SET-2 and HEL) [34] thus offering a potential means to circumvent thrombocytopenia associated with BCL- $\mathrm{X}_{\mathrm{L}}$ inhibition [34, 48, 49].

The diverse BCL-2 family expression profiles and differential sensitivities of NHL and AML to selective inhibitors of the BCL-2 family [17, 19, 34, 35] typify the heterogeneity associated with these hematopoietic neoplasms [37, 50]. TP53 mutation status did not impact the activity of A-1592668 in E $\mu$-myc lymphoma cells or AML cell lines (Supplementary Table 1) in vitro, aligning with 
observations obtained with dinaciclib [23, 51] and flavopiridol [52]. Leukemias driven by mixed lineage leukemia fusion proteins (MLL-FPs) that recruit CDK9-containing SECs are preferentially sensitive to pan-CDK inhibitors such as dinaciclib [53]. A-1592668 was active in AML cell lines harboring MLL-FPs either as a monotherapy (MV411, MLL-AF4) or in combination with venetoclax (THP-1; MLL-AF9). Further, FLT3-ITD AML has been identified as an AML subgroup where venetoclax is less active [40]. Reinforcing this clinical observation, venetoclax treatment was not active in a FLT3-ITD AML PDX model, providing a median survival benefit that was less than that observed with A-1592668 treatment alone and further highlighting the contribution of MCL-1 towards AML cell survival. Treatment of two AML PDX models with A-1592668 in combination with venetoclax significantly extended survival of tumor-bearing animals over those treated with either agent alone or the vehicle independent of the FLT3-ITD status. Thus, to drive deeper responses, circumvent disease heterogeneity, prevent clonal selection and/or to escape the generation of acquired resistance, targeting multiple antiapoptotic BCL-2 family members simultaneously is desired.

Collectively the data presented here demonstrate that potent CDK9 inhibitors such as A-1592668 downregulate MCL-1 expression to initiate apoptosis and tumor cell death in MCL-1-dependent hematologic tumors. Importantly, this mechanism drives combinatorial activity with the BCL-2 selective inhibitor venetoclax in a number of pre-clinical models of NHL and AML emphasizing the potential of CDK9 inhibitors in combination with venetoclax for the treatment of patients with hematologic malignancies.

Acknowledgements We thank the AbbVie DMPK team for their analytical support in determining rat PK parameters. RWJ is supported by a National Health and Medical Research Council (NHMRC) SPRF Fellowship, NHMRC project and program grants, the Cancer Council Victoria, the Kids Cancer Project and the Victorian Cancer Agency.

\section{Compliance with ethical standards}

Conflict of interest The design, study conduct, and financial support for this research were provided by AbbVie. AbbVie participated in the interpretation of data, review, and approval of this publication. DCP, SJ, JX, JC, YT, HZ, MS, SKT, RFC, TDP, DHA, JDL, and AJS are employees of AbbVie Inc. and are stock holders. GPG: Advisory board Takeda; speaker's honoraria from Roche; research support from Amgen, Merck and AbbVie Inc.JS: Advisory boards, Celgene, Novartis, and BMS; speaker's honoraria from Novartis, BMS, Roche, Amgen, and Takeda; research support from Amgen and Astex. RWJ: Research support from Abbvie Inc., Astra-Zeneca, BMS, Roche, and MecRX. MK: Advisory boards, F. Hoffman La-Roche and AbbVie; honoraria from Amgen, AbbVie, and Genentech; research funding from AbbVie, Genentech, Eli Lilly, Cellectis, Calithera, Stemline, Threshold, and Flexus; Reata Pharmaceuticals stock holder. EDH: Advisory board honoraria from Seattle Genetics and Celgene; research support from AbbVie Inc. and Eli Lilly. QZ, XZ, and JRD have nothing to disclose.
Publisher's note Springer Nature remains neutral with regard to jurisdictional claims in published maps and institutional affiliations.

\section{References}

1. Shapiro GI. Cyclin-dependent kinase pathways as targets for cancer treatment. J Clin Oncol. 2006;24:1770-83.

2. Hanahan D, Weinberg RA. Hallmarks of cancer: the next generation. Cell. 2011;144:646-74.

3. Morales F, Giordano A. Overview of CDK9 as a target in cancer research. Cell Cycle. 2016;15:519-27.

4. Lin TS, Ruppert AS, Johnson AJ, Fischer B, Heerema NA, Andritsos LA, et al. Phase II study of flavopiridol in relapsed chronic lymphocytic leukemia demonstrating high response rates in genetically high-risk disease. J Clin Oncol. 2009;27:6012-8.

5. Lanasa MC, Andritsos L, Brown JR, Gabrilove J, Caligaris-Cappio F, Ghia P, et al. Final results of EFC6663: a multicenter, international, phase 2 study of alvocidib for patients with fludarabine-refractory chronic lymphocytic leukemia. Leuk Res. 2015;39:495-500.

6. Holkova B, Perkins EB, Ramakrishnan V, Tombes MB, Shrader E, Talreja N, et al. Phase I trial of bortezomib (PS-341; NSC 681239) and alvocidib (flavopiridol; NSC 649890) in patients with recurrent or refractory B-cell neoplasms. Clin Cancer Res. 2011;17:3388-97.

7. Jones JA, Rupert AS, Poi M, Phelps MA, Andritsos L, Baiocchi $\mathrm{R}$, et al. Flavopiridol can be safely administered using a pharmacologically derived schedule and demonstrates activity in relapsed and refractory non-Hodgkin's lymphoma. Am J Hematol. 2014;89:19-24.

8. Jonkers I, Lis JT. Getting up to speed with transcription elongation by RNA polymerase II. Nat Rev Mol Cell Biol. 2015;16:167-77.

9. Peterlin BM, Price DH. Controlling the elongation phase of transcription with P-TEFb. Mol Cell. 2006;23:297-305.

10. Moldoveanu T, Follis AV, Kriwacki RW, Green DR. Many players in BCL-2 family affairs. Trends Biochem Sci. 2014;39:101-11.

11. Strasser A, Cory S, Adams JM. Deciphering the rules of programmed cell death to improve therapy of cancer and other diseases. EMBO J. 2011;30:3667-83.

12. Ashkenazi A, Fairbrother WJ, Leverson JD, Souers AJ. From basic apoptosis discoveries to advanced selective BCL-2 family inhibitors. Nat Rev Drug Discov. 2017;16:273-84.

13. Souers AJ, Leverson JD, Boghaert ER, Ackler SL, Catron ND, Chen J, et al. ABT-199, a potent and selective BCL-2 inhibitor, achieves antitumor activity while sparing platelets. Nat Med. 2013;19:202-8.

14. Marschitz I, Tinhofer I, Hittmair A, Egle A, Kos M, Greil R. Analysis of $\mathrm{Bcl}-2$ protein expression in chronic lymphocytic leukemia. A comparison of three semiquantitation techniques. Am J Clin Pathol. 2000;113:219-29.

15. Robertson LE, Plunkett W, McConnell K, Keating MJ, McDonnell TJ. Bcl-2 expression in chronic lymphocytic leukemia and its correlation with the induction of apoptosis and clinical outcome. Leukemia. 1996;10:456-9.

16. Roberts AW, Davids MS, Pagel JM, Kahl BS, Puvvada SD, Gerecitano JF, et al. Targeting BCL2 with venetoclax in relapsed chronic lymphocytic leukemia. N Engl J Med. 2016;374:311-22.

17. Phillips DC, Xiao Y, Lam LT, Litvinovich E, Roberts-Rapp L, Souers AJ, et al. Loss in MCL-1 function sensitizes non-Hodgkin's lymphoma cell lines to the BCL-2-selective inhibitor venetoclax (ABT-199). Blood Cancer J. 2015;5:e368.

18. Tahir SK, Smith ML, Hessler P, Rapp LR, Idler KB, Park CH, et al. Potential mechanisms of resistance to venetoclax and strategies to circumvent it. BMC Cancer. 2017;17:399.

19. Li L, Pongtornpipat P, Tiutan T, Kendrick SL, Park S, Persky DO, et al. Synergistic induction of apoptosis in high-risk DLBCL by 
BCL2 inhibition with ABT-199 combined with pharmacologic loss of MCL1. Leukemia. 2015;29:1702-12.

20. Booher RN, Hatch H, Dolinski BM, Nguyen T, Harmonay L, Al-Assaad AS, et al. MCL1 and BCL-xL levels in solid tumors are predictive of dinaciclib-induced apoptosis. PloS ONE. 2014;9: e108371.

21. Chen R, Keating MJ, Gandhi V, Plunkett W. Transcription inhibition by flavopiridol: mechanism of chronic lymphocytic leukemia cell death. Blood. 2005;106:2513-9.

22. Gojo I, Zhang B, Fenton RG. The cyclin-dependent kinase inhibitor flavopiridol induces apoptosis in multiple myeloma cells through transcriptional repression and down-regulation of Mcl-1. Clin Cancer Res. 2002;8:3527-38.

23. Gregory GP, Hogg SJ, Kats LM, Vidacs E, Baker AJ, Gilan O, et al. CDK9 inhibition by dinaciclib potently suppresses Mcl-1 to induce durable apoptotic responses in aggressive MYC-driven Bcell lymphoma in vivo. Leukemia. 2015;29:1437-41.

24. Xiao Y, Nimmer P, Sheppard GS, Bruncko M, Hessler P, Lu X, et al. MCL-1 is a key determinant of breast cancer cell survival: validation of MCL-1 dependency utilizing a highly selective small molecule inhibitor. Mol Cancer Ther. 2015;14:1837-47.

25. Rowland T, Paiva C, Rowley J, Chen A, Drew L, Hurlin P, et al. Selective targeting cyclin-dependent kinase-9 (CDK9) downmodulates c-MYC and induces apoptosis in diffuse large B-Cell lymphoma (DLBCL) cells. Blood. 2016;128:289.

26. Garriga J, Xie H, Obradovic Z, Grana X. Selective control of gene expression by CDK9 in human cells. J Cell Physiol. 2010;222:200-8.

27. Dey J, Deckwerth TL, Kerwin WS, Casalini JR, Merrell AJ, Grenley MO, et al. Voruciclib, a clinical stage oral CDK9 inhibitor, represses MCL-1 and sensitizes high-risk diffuse large Bcell lymphoma to BCL2 inhibition. Sci Rep. 2017;7:18007.

28. Flinn IW, Byrd JC, Bartlett N, Kipps T, Gribben J, Thomas D, et al. Flavopiridol administered as a 24-hour continuous infusion in chronic lymphocytic leukemia lacks clinical activity. Leuk Res. 2005;29:1253-7.

29. Lin TS, Howard OM, Neuberg DS, Kim HH, Shipp MA. Seventytwo hour continuous infusion flavopiridol in relapsed and refractory mantle cell lymphoma. Leuk Lymphoma. 2002;43:793-7.

30. Nemunaitis JJ, Small KA, Kirschmeier P, Zhang D, Zhu Y, Jou YM, et al. A first-in-human, phase 1, dose-escalation study of dinaciclib, a novel cyclin-dependent kinase inhibitor, administered weekly in subjects with advanced malignancies. J Transl Med. 2013;11:259.

31. Hogg SJ, Newbold A, Vervoort SJ, Cluse LA, Martin BP, Gregory GP, et al. BET inhibition induces apoptosis in aggressive B-cell lymphoma via epigenetic regulation of BCL-2 family members. Mol Cancer Ther. 2016;15:2030-41.

32. Berenbaum MC. Criteria for analyzing interactions between biologically active agents. Adv Cancer Res. 1981;35:269-335.

33. Borisy AA, Elliott PJ, Hurst NW, Lee MS, Lehár J, Price ER, et al. Systematic discovery of multicomponent therapeutics. Proc Natl Acad Sci USA. 2003;100:7977-82.

34. Leverson JD, Phillips DC, Mitten MJ, Boghaert ER, Diaz D, Tahir SK, et al. Exploiting selective BCL-2 family inhibitors to dissect cell survival dependencies and define improved strategies for cancer therapy. Sci Transl Med. 2015;7:279ra40.

35. Kotschy A, Szlavik Z, Murray J, Davidson J, Maragno AL, Le Toumelin-Braizat G, et al. The MCL1 inhibitor S63845 is tolerable and effective in diverse cancer models. Nature. 2016;538:477-82.

36. Teh TC, Nguyen NY, Moujalled DM, Segal D, Pomilio G, Rijal $\mathrm{S}$, et al. Enhancing venetoclax activity in acute myeloid leukemia by co-targeting MCL1. Leukemia. 2018;32:303-12.
37. Li S, Mason CE, Melnick A. Genetic and epigenetic heterogeneity in acute myeloid leukemia. Curr Opin Genet Dev. 2016;36:100-6.

38. Konopleva M, Pollyea DA, Potluri J, Chyla B, Hogdal L, Busman $\mathrm{T}$, et al. Efficacy and biological correlates of response in a phase II study of venetoclax monotherapy in patients with acute myelogenous leukemia. Cancer Discov. 2016;6:1106-17.

39. DiNardo CD, Pratz KW, Letai A, Jonas BA, Wei AH, Thirman M, et al. Safety and preliminary efficacy of venetoclax with decitabine or azacitidine in elderly patients with previously untreated acute myeloid leukaemia: a non-randomised, open-label, phase $1 \mathrm{~b}$ study. Lancet Oncol. 2018;19:216-28.

40. Popovic R, Daver N, Ruvolo V, Chen K, Wang Z, Huang X, et al. Abstract CT069: correlative biomarkers of clinical activity of the BCL-2 inhibitor, venetoclax (ABT-199/ GDC-0199), in acute myeloid leukemia patients. Cancer Res. 2016;76(14 Supplement): CT069-CT.

41. Glaser SP, Lee EF, Trounson E, Bouillet P, Wei A, Fairlie WD, et al. Anti-apoptotic Mcl-1 is essential for the development and sustained growth of acute myeloid leukemia. Genes Dev. 2012;26:120-5.

42. Kelly GL, Grabow S, Glaser SP, Fitzsimmons L, Aubrey BJ, Okamoto T, et al. Targeting of MCL-1 kills MYC-driven mouse and human lymphomas even when they bear mutations in $\mathrm{p} 53$. Genes Dev. 2014;28:58-70.

43. Leverson JD, Zhang H, Chen J, Tahir SK, Phillips DC, Xue J, et al. Potent and selective small-molecule MCL-1 inhibitors demonstrate on-target cancer cell killing activity as single agents and in combination with ABT-263 (navitoclax). Cell Death Dis. 2015;6:e1590.

44. Davids MS, Roberts AW, Seymour JF, Pagel JM, Kahl BS, Wierda WG, et al. Phase I first-in-human study of venetoclax in patients with relapsed or refractory non-Hodgkin lymphoma. J Clin Oncol. 2017;35:826-33.

45. Bodo J, Zhao X, Durkin L, Souers AJ, Phillips DC, Smith MR, et al. Acquired resistance to venetoclax (ABT-199) in $\mathrm{t}(14 ; 18)$ positive lymphoma cells. Oncotarget. 2016;7:70000-10.

46. Stewart DP, Koss B, Bathina M, Perciavalle RM, Bisanz K, Opferman JT. Ubiquitin-independent degradation of antiapoptotic MCL-1. Mol Cell Biol. 2010;30:3099-110.

47. Leich E, Salaverria I, Bea S, Zettl A, Wright G, Moreno V, et al. Follicular lymphomas with and without translocation $\mathrm{t}(14 ; 18)$ differ in gene expression profiles and genetic alterations. Blood. 2009;114:826-34.

48. Zhang H, Nimmer PM, Tahir SK, Chen J, Fryer RM, Hahn KR, et al. Bcl-2 family proteins are essential for platelet survival. Cell Death Differ. 2007;14:943-51.

49. Mason KD, Carpinelli MR, Fletcher JI, Collinge JE, Hilton AA, Ellis S, et al. Programmed anuclear cell death delimits platelet life span. Cell. 2007;128:1173-86.

50. Horn H, Staiger AM, Ott G. New targeted therapies for malignant lymphoma based on molecular heterogeneity. Exp Rev Hematol. 2017;10:39-51.

51. Chen Y, Germano S, Clements C, Samuel J, Shelmani G, Jayne S, et al. Pro-survival signal inhibition by CDK inhibitor dinaciclib in chronic lymphocytic leukaemia. Br J Haematol. 2016;175:641-51.

52. Parker BW, Kaur G, Nieves-Neira W, Taimi M, Kohlhagen G, Shimizu T, et al. Early induction of apoptosis in hematopoietic cell lines after exposure to flavopiridol. Blood. 1998;91:458-65.

53. Baker A, Gregory GP, Verbrugge I, Kats L, Hilton JJ, Vidacs E, et al. The CDK9 inhibitor dinaciclib exerts potent apoptotic and antitumor effects in preclinical models of MLL-rearranged acute myeloid leukemia. Cancer Res. 2016;76:1158-69. 